Christian Carow, Frank Rackwitz, Stavros Savidis

\title{
Über ein elastoplastisches Stoffgesetz für zyklisch beanspruchten Sand
}

Journal article | Accepted manuscript (Postprint)

This version is available at https://doi.org/10.14279/depositonce-9298

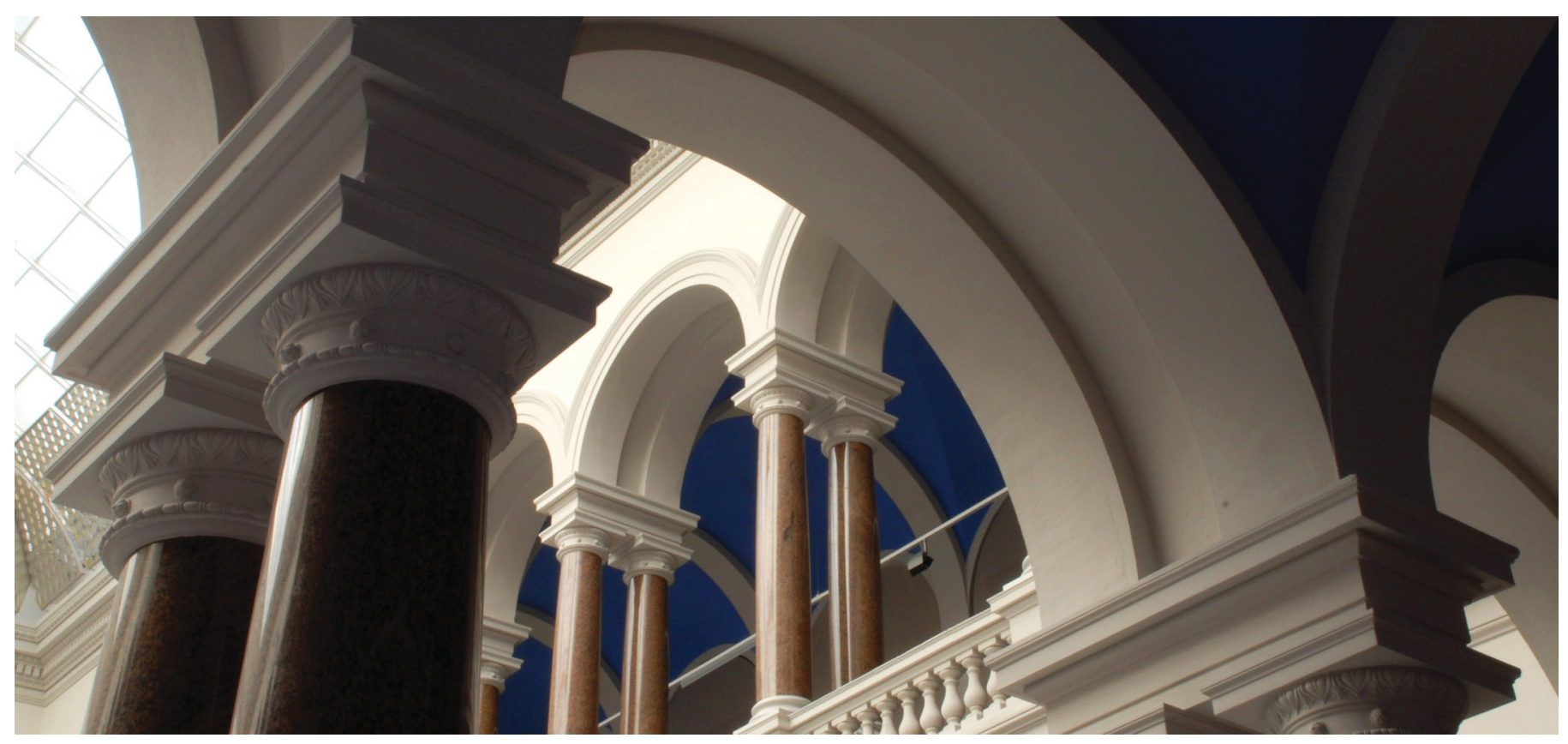

This is the pre-peer reviewed version of the following article / Dies ist die angenommene Version des folgenden Artikels:

Carow, C., Rackwitz, F., \& Savidis, S. (2017). Über ein elastoplastisches Stoffgesetz für zyklisch beanspruchten Sand. Bautechnik, 94(9), 604-612. https://doi.org/10.1002/bate.201700042

which has been published in final form at / die in endgültiger Form veröffentlicht wurde unter https://doi. org/10.1002/bate.201700042.

This article may be used for non-commercial purposes in accordance with Wiley Terms and Conditions for Use of Self-Archived Versions. / Dieser Artikel darf für nicht-gewerbliche Zwecke entsprechend der Wiley Selbstarchivierungs-Richtlinie verwendet werden [olabout.wiley.com/WileyCDA/Section/id-820227.html].

\section{Terms of Use}

Copyright applies. A non-exclusive, non-transferable and limited right to use is granted. This document is intended solely for 
Christian Carow (Hauptautor)

Frank Rackwitz (Co-Autor 1)

Stavros Savidis (Co-Autor 2)

\section{Über ein elastoplastisches Stoffgesetz für zyklisch bean-}

\section{spruchten Sand}

Die numerische Simulation aktueller Problemstellungen in der Geotechnik erfordert Stoffgesetze, die das Spannungs-Dehnungs-Verhalten von Sand realitätsnah beschreiben. In Bezug auf monotone Lasten sind mit bestehenden Modellierungsansätzen bereits sehr gute Ergebnisse zu erzielen. Das Materialverhalten bei zyklischer Beanspruchung ist jedoch außerordentlich komplex. Selbst die derzeit leistungsfähigsten Stoffgesetze können nur einzelne Aspekte abbilden. Daher ist weitere Forschungsarbeit dringend erforderlich.

Vor diesem Hintergrund stellt der Beitrag ein Stoffgesetz für Sand vor, das auf der Grenzflächenplastizität sowie dem Konzept kritischer Zustände basiert. Es kann das Verhalten des Bodens für komplexe zyklische Belastungspfade über eine breite Spanne von Dichte- und Spannungszuständen mit einem einzigen Parametersatz abbilden. Die Funktionsweise des Stoffgesetzes wird im Beitrag konzeptionell dargestellt. Die Ergebnisse der numerischen Simulation zyklischer undränierter Triaxialversuche werden den Ergebnissen entsprechender Laborversuche gegenübergestellt. Der Vergleich liefert eine gute Übereinstimmung. Die Eignung des Stoffgesetzes für den Einsatz in komplexen numerischen Simulationen wird anhand eines Berechnungsbeispiels gezeigt. Hierfür wurde ein Staudamm gewählt, der durch einen Erdbebenzeitverlauf an der Basis angeregt wird. Abschließend werden im Beitrag Ansätze zur Weiterentwicklung des Stoffgesetzes aufgezeigt.

Keywords Soffgesetze; Sand; Grenzflächenplastizität, zyklische Belastung

\section{Modeling the behavior of cyclically loaded sands by means of a bounding surface plasticitiy} model Thanks to contemporary information processing technology it is possible to numerically solve complicated geotechnical boundary value problems that involve cyclic loading. In order to obtain reliable and realistic simulation results one has to utilize an efficient and powerful constitutive model for the soil. 
Vast amounts of such models have been developed during the past five decades. Nevertheless, a sufficiently comprehensive solution is still missing, especially with regard to sands.

This article aims at presenting a model for sands which currently seems to be one of the most promising. The model is based on the principles of bounding surfaces plasticity and critical state soil mechanics. With a single set of material constants, the model is able to reproduce the stress strain behavior of sands over a broad range of stresses and densities for monotonic as well as for cyclic load paths. The article depicts the modeling approach in detail. The capabilities of the model are illustrated by simulating cyclic laboratory tests and the response of a reservoir dam to earthquake loading. After that the models shortcomings are analyzed and further developments are being discussed.

Keywords constitutive models; sands; bounding surface plasticity; cyclic loading

\section{$1 \quad$ Einleitung}

Numerische Simulationen mit der Finite-Elemente-Methode sind in der Geotechnik schon seit Langem ein unentbehrliches Werkzeug zur Lösung praktischer Bemessungsaufgaben. Dank der rasanten Entwicklung der Rechentechnik können zunehmend auch solche Anfangsrandwertprobleme gelöst werden, bei denen zyklische Beanspruchungen des Baugrundes auftreten. Entsprechende Aufgabenstellungen ergeben sich beispielsweise aus Standsicherheits- und Gebrauchstauglichkeitsuntersuchungen im Offshore-Bereich, in Erdbebenregionen und im Verkehrswegebau. Zudem gibt es Bestrebungen, geotechnische Herstellungsprozesse, die mit zyklischen Vorgängen wie Rammen, Rütteln und Vibrieren einhergehen, in numerischen Prognosemodellen zu berücksichtigen [1].

Um bei FE-Berechnungen in der Geotechnik realitätsnahe Ergebnisse erzielen zu können, werden leistungsfähige Stoffgesetze für das Spannungs-Dehnungs-Verhalten des Baugrundes benötigt. Aufgrund experimenteller Untersuchungen ist bekannt, dass die Reaktion von Sand auf eine äußere Einwirkung mindestens von den folgenden Faktoren abhängig ist: Aktueller Spannungs- und Dichtezustand, Beanspruchungsgeschichte, inhärente und induzierte Anisotropie der Kornstruktur, Entwässerungsbedingungen, Richtung und Größe der Last. Daraus ergeben sich für die Materialmodellierung eine ganze Reihe von Herausforderungen. Diese werden nochmals massiv vergrößert, sofern zyklische Belastungen berücksichtigt werden sollen.

Vor diesem Hintergrund wird im Folgenden der Stand der Forschung hinsichtlich der Stoffmo- 
dellierung bei Sand diskutiert. Im Fokus liegt dabei der Bereich kleiner bis großer Scherdehnungen $\left(\gamma>10^{-3}\right)$ und ein- bis zweistelliger Zyklenzahlen $(N<50)$. Spezielle Modellierungsansätze für sehr kleine Dehnungen, hochzyklische Vorgänge oder Spannungsniveaus, bei denen mit Kornbruch in signifikantem Ausmaß zu rechnen ist, werden nicht betrachtet.

Im Ergebnis der Diskussion wird ein Stoffgesetz ausgewählt, welches das Potential bietet, das zyklische Spannungs-Dehnungs-Verhaltens von Sand vergleichsweise umfassend zu beschreiben. Dieses Stoffgesetz wird anschließend näher vorgestellt. Seine Anwendung wird anhand eines Berechnungsbeispiels demonstriert. Zum Schluss werden Ansätze zur Weiterentwicklung des Stoffgesetzes aufgezeigt.

\section{2 Überblick bestehender Modellierungsansätze}

Stoffgesetze für nichtbindige Böden sind seit Jahrzehnten Gegenstand vieler Forschungsarbeiten. Mittlerweile sind daraus einige leistungsfähige Modellierungsansätze entstanden.

Insbesondere im deutschsprachigen Raum hat sich das hypoplastische Stoffgesetz mit der intergranularen Dehnung weithin etabliert [2], [3]. Seine Anwendung auf geotechnische Probleme mit zyklischen Lasten wurde bereits ausführlich dokumentiert, bspw. in [4], [5]. Es wird zudem laufend verbessert [6-8].

Eine zentrale Bedeutung kommt bei der Stoffmodellierung für granulare Stoffe der Berücksichtigung des Dilatanzverhaltens zu. Unter Dilatanz versteht man Volumenänderungen, die sich als Folge der Scherverformungen des Korngerüstes ergeben. Bei nichtbindigen Böden hängt die Dilatanz sowohl von der Spannung als auch von der Porenzahl ab. Die Stoffgesetzfunktionen, die in der Hypoplastizität die Dilatanz regulieren, sind jedoch nur von der Spannung abhängig. Insofern bestehen bei der Hypoplastizität noch Unwägbarkeiten bezüglich der Prognose von Volumendehnungen beziehungsweise Porenwasserüberdrücken, die durch Scherung ausgelöst werden.

Der Abbildung des Dilatanzverhaltens nichtbindiger Böden wurde im Bereich der Elastoplastizität ebenfalls besondere Aufmerksamkeit gewidmet. Prägend war die Arbeit von Li und Dafalias [9], worin ein Dilatanzmodell für Sand vorgestellt wurde. Es prognostiziert schubinduzierte Volumenänderungen sowohl in Abhängigkeit von der mittleren effektiven Spannung $p^{\prime}$ als auch von der Porenzahl $e$. Der Materialzustand des Sandes wird mit Hilfe der Critical State 
Line (CSL) bewertet. Die CSL ist eine Kurve im $p^{\prime}-e$-Raum, die ein Sand nach großen Scherwegen erreicht. Sie wird in der Tradition der „Critical State Soil Mechanics“ als intrinsische Materialeigenschaft angesehen [10].

Aufbauend auf [9] entwickelte Li [11] ein Stoffgesetz, das hier wegen des Bezugs zu den Critical State Soil Mechanics als „Critical State Sand Model“ (CSSA-Modell) bezeichnet wird. Es basiert auf der Theorie der Grenzflächenplastizität (engl. bounding surface plasticity) [12]. Damit eignet es sich sehr gut für den Einsatz bei der Lösung von Anfangsrandwertproblemen mit zyklischen Lasten [13], [14]. Zudem wird es kontinuierlich weiterentwickelt. Die neueste Entwicklungsstufe des Modells ist in der Lage, auch die Entwicklung der inhärent anisotropen Kornstruktur von Sand unter zyklischer Beanspruchung abzubilden [15]. Dies gelingt, indem sowohl der Verfestigungsmodul als auch die Dilatanz als Funktionen eines dreidimensionalen Gefügetensors (engl. fabric tensor) formuliert werden, der als zusätzliche Zustandsvariable die anisotrope Kornstruktur kontinuumsmechanisch beschreibt.

Das Dilatanzmodell von Li und Dafalias [9] erfuhr breite Resonanz und wurde in weitere elastoplastische Stoffgesetze integriert. So fand es auch Eingang in das populäre SANISAND-Modell [16]. Neben dem Dilatanzmodell sind die Kernkomponenten von SANISAND eine Fließfläche, eine Grenzfläche und die sogenannte Critical-State-Fläche. Durch Rotation der Fließfläche um den Ursprung des Spannungsraumes kann spannungsinduzierte Anisotropie abgebildet werden. Diese kinematische Verfestigung wird mittels der Grenzflächenplastizität gesteuert. Die Critical-State-Fläche hingegen stellt die äußere Begrenzung aller möglichen Materialzustände dar. Mit diesem Ansatz kann SANISAND die Antwort des Bodens auf zyklische Beanspruchungen sehr gut wiedergeben, wie beispielsweise Taiebat u. a. [17] demonstrieren. Die SANISANDTheorie wurde daher in einer ganzen Reihe von Arbeiten weiterentwickelt (bspw. [18], [19]), und auch mit der Hypoplastizität kombiniert [6], [20].

Es existiert eine große Zahl weiterer Stoffgesetze für Sand. Ihnen gegenüber nehmen die Hypoplastizität, SANISAND und das CSSA-Modell jedoch eine Vorreiterrolle ein. Sie weisen eine sehr lange Geschichte kontinuierlicher Weiterentwicklung auf und sind daher mittlerweile in der Lage, vergleichsweise viele Aspekte des Materialverhaltens unter zyklischer Beanspruchung abzubilden. Zudem wurden sie sorgfältig durch Nachrechnung von Elementversuchen und Benchmark-Problemen validiert. Welcher der drei genannten Modellierungsansätze letztlich der absolut beste ist, kann nicht eindeutig festgestellt werden. Jeder weist spezifische Stärken und 
Schwächen auf und hat somit seine eigene Existenzberechtigung.

Das CSSA-Modell hat gegenüber den meisten anderen elastoplastischen Modellen für nichtbindige Böden einen konzeptionellen Vorteil. Es kann auch inelastische Verformungen prognostizieren, die direkt aus Änderungen der mittleren effektiven Spannung $p$ resultieren. Dies ermöglicht eine vollständige Beschreibung des Spannungs-Dehnungs-Verhaltens. Auch SANISAND wurde mit einer vergleichbaren Funktion ausgestattet [21], sie scheint jedoch numerische Probleme zu verursachen [22]. Gegenüber der Hypoplastizität besteht ein Vorteil des CSSA-Modells hinsichtlich der Modellierung des Dilatanzverhaltens. Es lohnt sich folglich, das CSSA-Modell dem deutschsprachigen Fachpublikum eingehend vorzustellen und Ansätze zur Weiterentwicklung zu suchen. Dem widmen sich die nächsten Abschnitte des Beitrages. Hierbei wird zugunsten einer zugänglichen Darstellung bewusst darauf verzichtet, den Formelapparat von $\mathrm{Li}[11]$ im Detail wiederzugeben.

\section{Critical State Stoffgesetz für Sand nach Li (CSSA-Modell)}

\subsection{Grundkonzept}

Das CSSA-Modell ist ein elastoplastisches Stoffgesetz vom Ratentyp [11]. Es hat die Form

$$
\dot{\sigma}^{\prime}=\mathrm{c}^{\mathrm{ep}}: \dot{\boldsymbol{\varepsilon}}
$$

Darin verknüpft die elastoplastische Steifigkeit $\mathbf{c}^{\text {ep }}$ die Rate der infinitesimalen Verzerrung $\dot{\boldsymbol{\varepsilon}}$ mit der Rate der effektiven Spannung $\dot{\sigma}^{\prime}$. Der Ausgangspunkt für die Formulierung der Gleichung für die Steifigkeit ist die additive Aufspaltung

$$
\dot{\boldsymbol{\varepsilon}}=\dot{\boldsymbol{\varepsilon}}^{\mathrm{e}}+\dot{\boldsymbol{\varepsilon}}^{\mathrm{p}}
$$

der Verzerrungsrate in einen reversiblen (elastischen) Anteil $\dot{\boldsymbol{\varepsilon}}^{\mathrm{e}}$ und einen irreversiblen (plastischen) Anteil $\dot{\varepsilon}^{\mathrm{p}}$. Der elastische Anteil korrespondiert mit der Spannungsrate über eine elastische Spannungs-Dehnungs-Beziehung.

Zur Berechnung des plastischen Anteils $\dot{\boldsymbol{\varepsilon}}^{p}$ wird die Spannungsrate gemäß der Kettenregel der Differentiation in zwei Anteile aufgeteilt:

$$
\dot{\boldsymbol{\sigma}}^{\prime}=\dot{\boldsymbol{r}} p+\dot{p}\left(\boldsymbol{\sigma}^{\prime} / p\right)
$$

Darin ist $\boldsymbol{r}=\boldsymbol{\sigma}_{\mathrm{dev}}^{\prime} / p$ der Tensor des Spannungsverhältnisses. Plastische Verformungen infolge $\dot{\boldsymbol{r}}$ werden beim CSSA-Modell mittels einer kegelförmigen Grenzfläche berechnet, die 
Abbildung 1 zeigt. Der Kegel wird senkrecht zur hydrostatischen Achse durch die zweite Grenzfläche, eine flache Kappe, abgeschlossen. Sie dient dazu, plastische Verzerrungen zu berechnen, die aus Änderungen der mittleren effektiven Spannung bei konstantem Spannungsverhältnis resultieren, also dem Anteil $\dot{p}$ in (3).

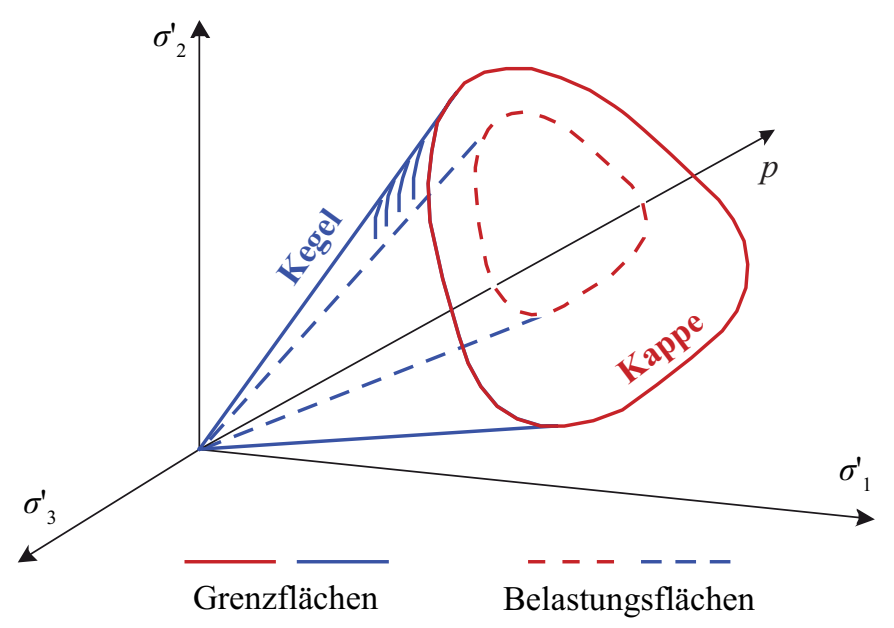

Abbildung 1: Schematische Illustration der Grenz- und Belastungsflächen des CSSA-Modells im Hauptspannungsraum

Beide Grenzflächen verfestigen unter monotoner Beanspruchung isotrop, wobei laufend sowohl elastische als auch plastische Verformungen produziert werden. Bei zyklischer Be- und Entlastung liegt der aktuelle Spannungspunkt im Inneren der Grenzflächen auf dem Schnittpunkt zweier sogenannter Belastungsflächen. Mittels Abbildungsregeln wird der Spannungspunkt auf die Grenzflächen projiziert. Anhand des Abstandes des Spannungspunktes von seinem Abbild kann der Einfluss der zyklischen Vorbelastungsgeschichte auf die Materialsteifigkeit eingeschätzt werden. Hierin liegt der maßgebliche Vorteil der Grenzflächenplastizität gegenüber anderen Modellierungsansätzen wie der klassischen Plastizitätstheorie oder der Hypoplastizität im Hinblick auf zyklische Beanspruchungen.

\subsection{Materialzustandsabhänige Dilatanz}

Neben der Grenzflächenplastizität ist der zweite integrale Bestandteil des Stoffgesetzes das Dilatanzmodell. Es reguliert die Volumenänderung des Bodens im Verhältnis zur Schubverzerrungsrate. Dafür wird der aktuelle Materialzustand sowohl bezüglich des Spannungsniveaus als auch der Dichte des Bodens bewertet. Als Referenz dient die CSL, die jeder mittleren effektiven Spannung $p$ eine Porenzahl im kritischen Zustand $e_{\mathrm{c}}$ zuordnet und als intrinsische Materialeigenschaft angesehen wird. Die kritische Porenzahl wird als 


$$
e_{c}=e_{\Gamma}-\lambda_{c}\left(p^{\prime /} p_{a}\right)^{\xi}
$$

beschrieben, worin $p_{a}$ ein Referenzdruck ist und $e_{\Gamma}, \lambda_{c}$ sowie $\xi$ Materialparameter sind. Die CSL nach (4) ist im $\left(p / p_{a}\right)^{\xi}-e$-Diagramm, das Abbildung 2 zeigt, eine Gerade. Den Abstand zur CSL erfasst der Zustandsparameter $\psi=e-e_{\mathrm{c}}$, dessen Konzept auf Been und Jefferies [23] zurückgeht. Befindet sich der aktuelle Materialzustand über der CSL (Punkt A in Abbildung 2), ist $\psi>0$ und der Boden wird unter Scherbeanspruchung verdichten. Das Modell berechnet folglich eine positive Volumendehnung. Im Fall $\psi<0$ (Punkt B in Abbildung 2) hingegen liegt ein dilatanter Zustand vor und das Modell reagiert auf Scherbeanspruchung mit Auflockerung.

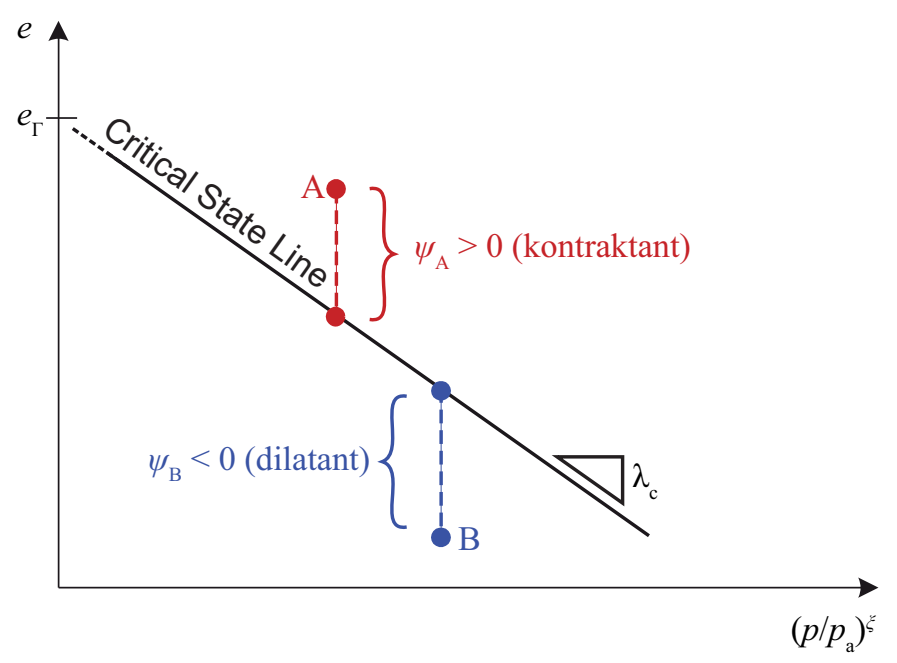

Abbildung 2: Critical State Line und Zustandsparameter $\psi$ im $\left(p / p_{a}\right)^{\xi}-e$-Diagramm

Der Zustandsparameter $\psi$ geht auch in die Verfestigungsregeln der Grenzflächen ein. Dies ermöglicht, das Verformungsverhalten des Bodens über einen sehr großen Bereich von Spannungen und Lagerungsdichten hinweg zu modellieren. Infolgedessen haben die Materialparameter konstante Werte für Porenzahlen zwischen $e_{\min }$ und $e_{\max }$ sowie für Drücke bis weit über $1000 \mathrm{kPa}[9]$.

\subsection{Materialparameter}

Das CSSA-Modell beinhaltet in der Version von Li [11] 15 dimensionslose Materialparameter, die sich in vier Gruppen aufteilen lassen, wie Tabelle 1 zeigt. Eine physikalische Bedeutung lässt sich den Parametern des elastischen Anteils und jenen des Critical State zuordnen. Die Bedeutung der anderen Parameter ergibt sich aus dem Kontext der Gleichungen des CSSAModells [9], [11]. 
Tabelle 1: Materialparameter des CSSA-Modells und deren Konstanten für Toyoura-Sand (alle Parameter sind dimensionslos)

\begin{tabular}{|c|c|c|c|}
\hline & Parameter & Wert & Bedeutung \\
\hline \multirow{2}{*}{ Elastischer Anteil } & $G_{0}$ & 125 & Schubsteifigkeit bei sehr kleinen Dehnungen \\
\hline & $v$ & 0,05 & Querdehnzahl \\
\hline \multirow{5}{*}{$\begin{array}{l}\text { Critical } \\
\text { State }\end{array}$} & $M_{\mathrm{c}}$ & 1,25 & Festigkeit im critical state (CS) bei triaxialer Kompression \\
\hline & $c$ & 0,7 & Verhältnis der Festigkeiten im CS bei triaxialer Extension/Kompression \\
\hline & $e_{\Gamma}$ & 0,934 & Porenzahl auf der CSL bei $p=0$ \\
\hline & $\lambda_{\mathrm{c}}$ & 0,019 & Steigung der CSL \\
\hline & $\xi$ & 0,7 & Exponent in der Gleichung für die CSL \\
\hline \multirow{6}{*}{$\begin{array}{c}\text { Kegel- } \\
\text { mechanismus }\end{array}$} & $d_{1}$ & 0,88 & Dilatanzparameter des Kegels \\
\hline & $m$ & 3,5 & Exponent in Dilatanzfunktion des Kegels \\
\hline & $h_{1}$ & 3,15 & Verfestigungsparameter des Kegels \\
\hline & $h_{2}$ & 3,05 & Verfestigungsparameter des Kegels \\
\hline & $h_{3}$ & 2,6 & Verfestigungsparameter des Kegels (zyklische Belastung) \\
\hline & $n$ & 1,1 & Exponent in Verfestigungsregel des Kegels \\
\hline \multirow{2}{*}{ Kappenmechanismus } & $d_{2}$ & 0,5 & Dilatanzparameter der Kappe \\
\hline & $h_{4}$ & 220 & Verfestigungsparameter der Kappe \\
\hline
\end{tabular}

Die Werte der Parameter $G_{0}$ und $v$ des elastischen Anteils ergeben sich aus Resonant-ColumnVersuchen. Die Querdehnzahl $v$ kann alternativ anhand der Ergebnisse undränierter Triaxialversuche kalibriert werden. Dadurch ergeben sich u. U. Werte, die außerhalb der üblichen Spanne von Versuchsergebnissen für $v$ liegen, wie bspw. auch beim Parametersatz in Tabelle 1. Die Parameter, die den Critical State betreffen (vgl. Abbildung 2), werden in dränierten und undränierten triaxialen Kompressions- und Extensionsversuchen bestimmt. An diesen Versuchen werden auch die Dilatanz- und Verfestigungsparameter des Kegelmechanismus kalibriert. Zusätzlich ist ein undränierter zyklischer Triaxialversuch erforderlich, um den Wert für den Parameter $h_{3}$ zu finden, der das Verhalten des Modells bei zyklischer Be- und Entlastung steuert. Die beiden Parameter der Kappe schließlich können anhand der Ergebnisse von Ödometerversuchen kalibriert werden.

Die Konstanten für Toyoura-Sand in Tabelle 1 wurden, bis auf den Wert für $h_{3}$, der Arbeit von Rackwitz [24] entnommen, in der auch die oben verkürzt dargestellte Prozedur ausführlich beschrieben wird. Auf die Ermittlung von $h_{3}$ wird hier in Abschnitt 4.2 näher eingegangen.

Der Aufwand bei der Parameterbestimmung erscheint vor dem Hintergrund einer praktischen 
Anwendung vergleichsweise hoch. Dies relativiert sich insofern, als die Stoffgesetzkonstanten beim CSSA-Modell nicht manuell an verschiedene Spannungsniveaus und Lagerungsdichten angepasst werden müssen, wie es bei weniger hoch entwickelten Stoffgesetzen der Fall ist.

\section{$4 \quad$ Berechnungsbeispiel}

\subsection{Problemstellung}

Um die Anwendung des CSSA-Modells auf ein Anfangsrandwertproblem zu demonstrieren, wurden Verformungsberechnungen für einen seismisch beanspruchten Staudamm durchgeführt. Das Finite-Elemente-Modell zeigt Abbildung 3. Die Modellierung erfolgte im Finite-ElementeProgrammsystem ANSYS 17.0. Die Geometrie ist angelehnt an den Unteren San Fernando Staudamm, der 1971 durch ein Erdbeben schwer beschädigt wurde [25]. Eine genaue Nachrechnung dieses Schadensfalles wird hier jedoch nicht angestrebt. Dafür müssten die Geometrie und die Materialparameter detailliert an die historischen Gegebenheiten angepasst werden.

Die horizontale Ausdehnung des Berechnungsausschnittes wurde so groß gewählt, dass die Wellenreflektionen an den seitlichen Modellrändern einen vernachlässigbaren Fehler verursachen. Der Verlauf der Sickerlinie wurde in einer stationären Strömungsberechnung ermittelt und dem Modell als Randbedingung eingeprägt. Der Einfluss des Porenwassers im Bereich unterhalb der Sickerlinie wird durch ein benutzerdefiniertes Zweiphasenelement berücksichtigt [26]. Der untere Modellrand, der den anstehenden Felshorizont repräsentiert, ist unverschieblich und undurchlässig. Über ihn wird das Erdbebensignal als Zeitverlauf einer horizontalen Verschiebung gemäß Abbildung 4 eingeleitet. An den seitlichen Modellrändern fungiert der hydrostatische Wasserdruck als Strömungsrandbedingung. Die horizontale Verschiebung der jeweils gegenüberliegenden Randknoten wurde gekoppelt, um für die dynamische Laststufe Randbedingungen analog denen im Freifeld herzustellen. 


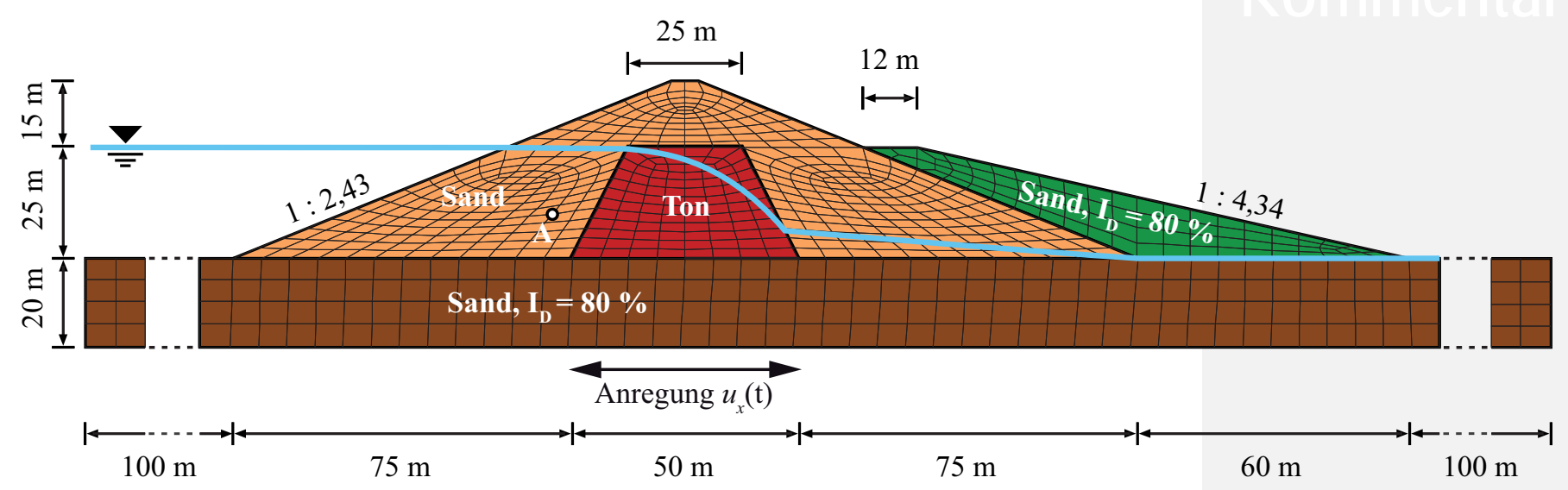

Abbildung 3: Finite-Elemente-Modell des Berechnungsbeispiels

Der Untergrund, die Dammschüttung und die luftseitige Berme bestehen aus Sand, dessen Spannungs-Dehnungs-Verhalten durch das CSSA-Modell mit den Parametern aus Tabelle 1 abgebildet wird. Die relative Lagerungsdichte $I_{\mathrm{d}}$ des Untergrundes und der Berme beträgt $80 \%$. Die Lagerungsdichte der Dammschüttung wurde variiert. Der Tonkern kann mit einem einfachen elastoplastischen Ansatz auf Basis der Bruchbedingung nach Drucker-Prager modelliert werden, weil bindige Böden aufgrund der Kohäsion vergleichsweise unempfindlich gegen seismisch induzierte Bodenverflüssigung sind. Die Scherparameter $\left(\phi=22^{\circ}, c=20 \mathrm{kN} / \mathrm{m}^{2}\right)$ entsprechen einem steifen Ton. Die Durchlässigkeiten der Böden im Modell wurden auf Grundlage typischer Erfahrungswerte wie folgt gewählt: Damm: $k_{1}=10^{-3} \mathrm{~m} / \mathrm{s}$, Untergrund: $k_{2}=10^{-4} \mathrm{~m} / \mathrm{s}$, Kern: $k_{3}=10^{-9} \mathrm{~m} / \mathrm{s}$. Vor allem der Wert für $k_{1}$ beeinflusst die Entwicklung der Verformungen infolge seismischer Anregung erheblich. Dies ließe sich durch eine umfangreiche Parameterstudie systematisch untersuchen, was jedoch nicht im Fokus des Beitrages liegt.

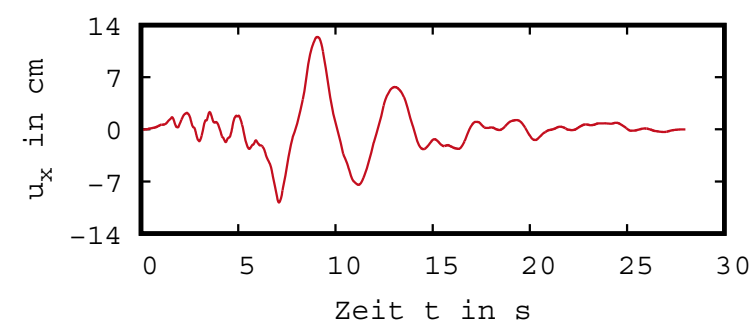

Abbildung 4: Verschiebungszeitverlauf des Anregungssignals für das Berechnungsbeispiel, abgeleitet aus Beschleunigungsaufzeichnungen des San Fernando Bebens von 1971 [27]

\subsection{Validierung des Stoffgesetzes}

Ein essentieller Arbeitsschritt bei numerischen Berechnungen mit einem komplexen Stoffgesetz besteht in der Validierung des verwendeten Parametersatzes. Der hier verwendete Parametersatz 
für Toyoura-Sand (Tabelle 1) wurde in Bezug auf monotone Beanspruchungen bereits umfassend validiert [11], [24]. Im Zusammenhang mit dem vorliegenden Aufsatz wurde nun auch der Parameter $h_{3}$ kalibriert, der die Entwicklung der Steifigkeit bei zyklischer Be- und Entlastung regelt. Hierfür wurden zyklische undränierte Triaxialversuche an Toyoura-Sand von Sze und Yang [28] mit dem CSSA-Modell nachgerechnet. Mit $h_{3}=2,6$ konnte das beste Simulationsergebnis erzielt werden. Dies veranschaulicht Abbildung 5. Gezeigt werden die Versuchsspur eines Wechsellastversuches und seine Nachrechnung mit dem CSSA-Modell. Der Ausgangsspannungszustand ist isotrop mit $p_{0}^{\prime}=100 \mathrm{kN} / \mathrm{m}^{2}$, die Porenzahl ist $e=0,717$.
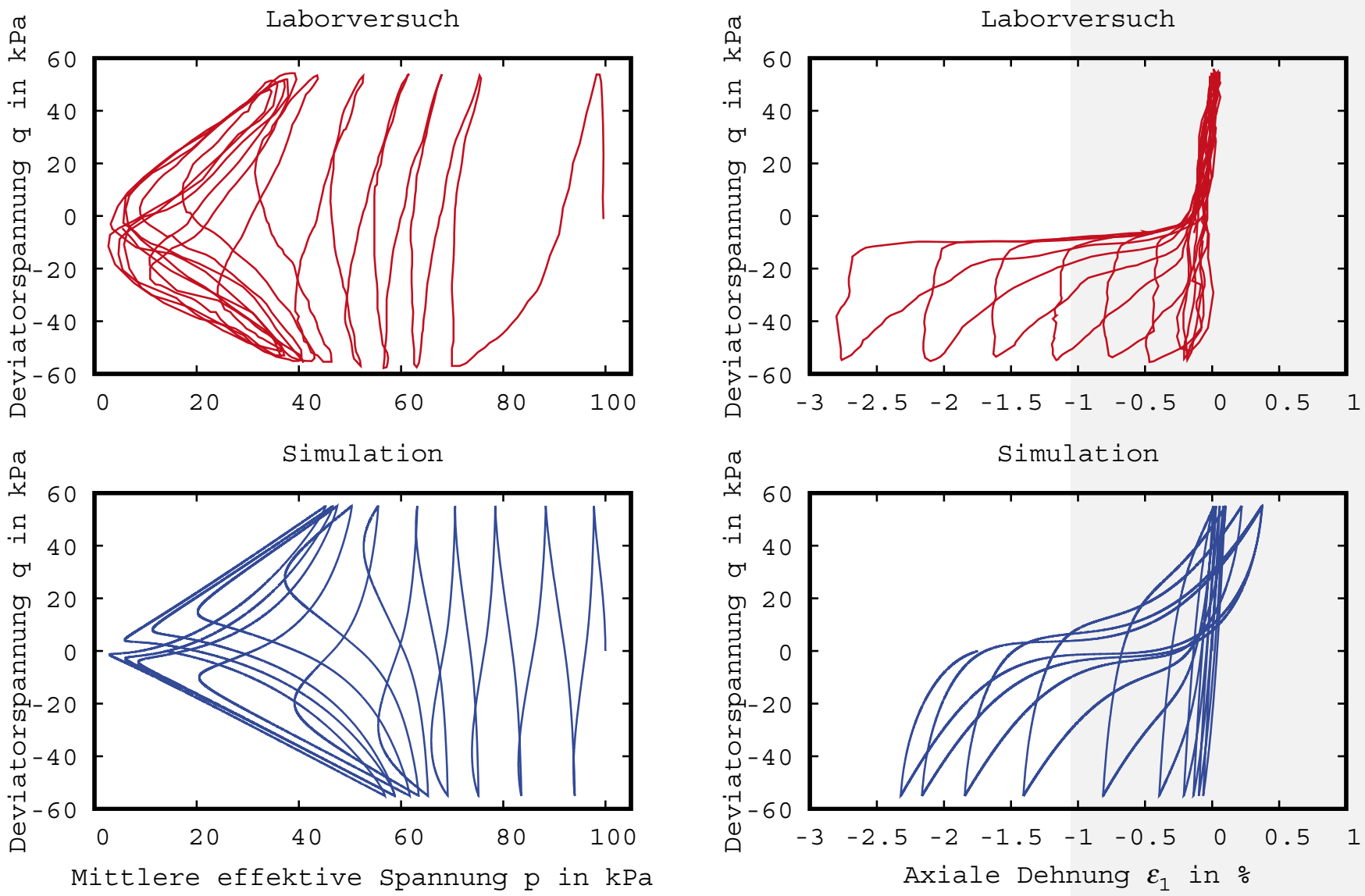

Abbildung 5: Versuchsergebnis (oben, aus [28]) und Nachrechnung (unten) eines undränierten zyklischen Triaxialversuches mit dem CSSA-Modell (Toyoura-Sand, Porenzahl $e=0,717$ )

Im Laborversuch nimmt der Porenwasserdruck durch die zyklische Scherung rapide zu, das effektive Spannungsniveau sinkt. Nach 6 Zyklen gerät die Probe in den Bereich der zyklischen Mobilität. Von da an verläuft der Spannungspfad in Schleifen um den Punkt $(p=0, q=0)$, während die Verformungen sukzessive zunehmen. Das Stoffgesetz kann dieses Verhalten mit dem gewählten Wert für den zyklischen Parameter $h_{3}$ sehr gut abbilden. Das Stoffgesetz und der 
Parametersatz können somit bei der numerischen Lösung von Anfangsrandwertproblemen mit zyklischen Lasten eingesetzt werden.

\subsection{Ergebnisse}

In Abbildung 6 und Abbildung 7 werden charakteristische Simulationsergebnisse für den Fall einer mitteldichten bzw. einer lockeren Lagerung der Dammschüttung gegenübergestellt. Bei mittlerer bezogener Lagerungsdichte $\left(I_{\mathrm{d}}=50 \%\right)$ liegt das Maximum der durch das Erdbeben verursachten Verschiebungen unmittelbar neben der Dammkrone (Abbildung 6 (a)). Die Setzung beträgt dort 1,4 m und die horizontale Verschiebung 1,6 m. Innerhalb der Dammschüttung kommt es zu einem moderaten Porenwasserdruckaufbau, wodurch das Niveau der effektiven Spannungen in Punkt A um etwa ein Drittel absinkt (Abbildung 7 (a)).

Dammschüttung mitteldicht gelagert

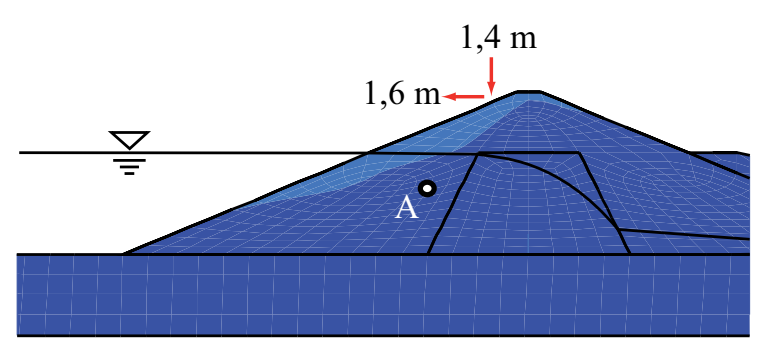

(a)
Dammschüttung locker gelagert

$|\boldsymbol{u}|$ in $\mathrm{m}$

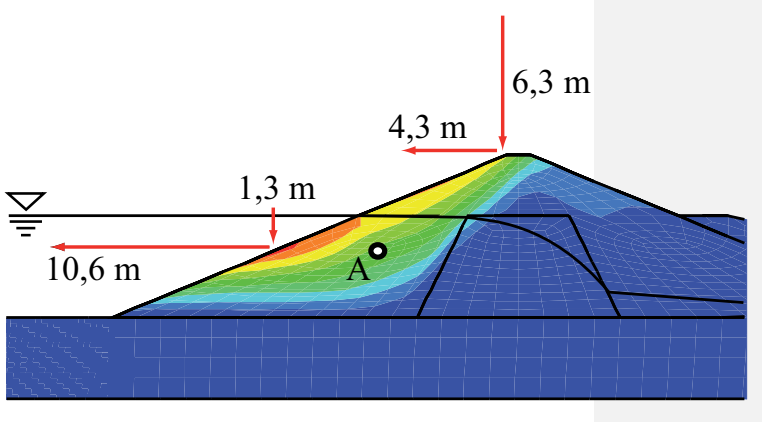

(b)

Abbildung 6: Intensität der Verschiebungen infolge der seismischen Anregung für den Fall einer mitteldicht gelagerten Dammschüttung mit $I_{\mathrm{d}}=50 \%$ (a) und den einer locker gelagerten Dammschüttung mit $I_{\mathrm{d}}=30 \%$ (b)

Dammschüttung mitteldicht gelagert

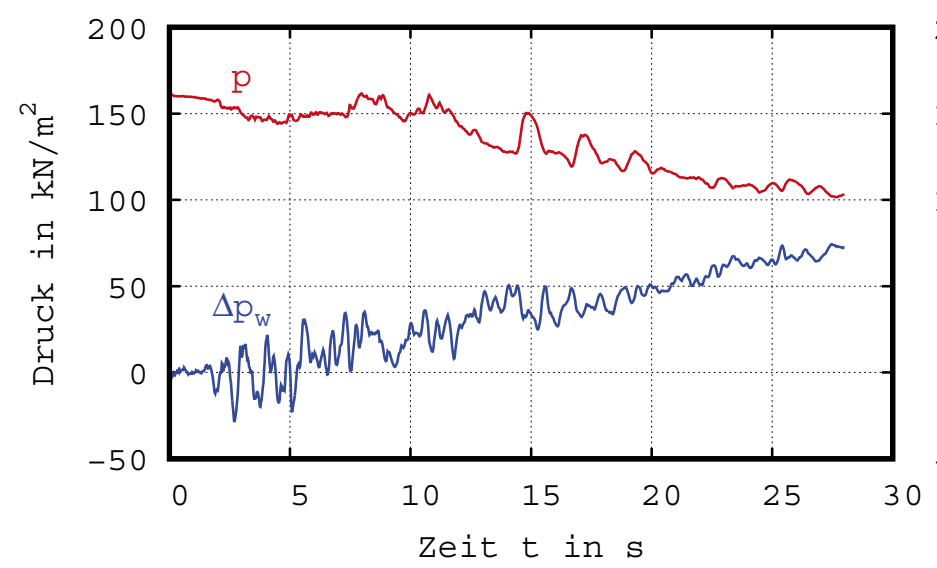

(a)
Dammschüttung locker gelagert

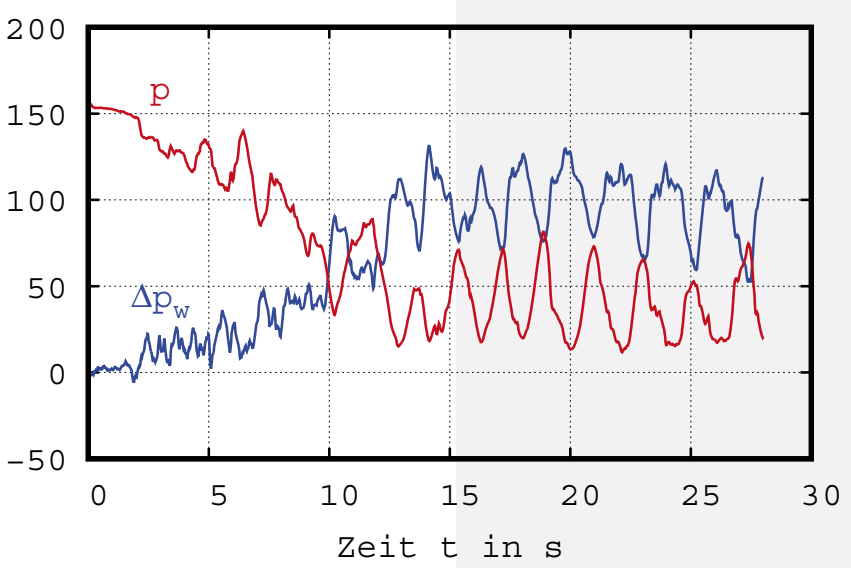

(b)

Abbildung 7: Zeitverlauf von mittlerer effektiver Spannung $p(t)$ und Änderung des Porenwasserdruckes $\Delta p_{\mathrm{W}}(t)=p_{\mathrm{W}}\left(t_{0}\right)-p_{\mathrm{W}}(t)$ im Punkt $\mathrm{A}$ während des Erdbebens für mitteldichte Lagerung (a) und lockere Lagerung (b) 
der Dammschüttung (zur Lage von Punkt A siehe Abbildung 3)

Bei lockerer Ausgangslagerungsdichte $\left(I_{\mathrm{d}}=30 \%\right)$ ergibt sich ein völlig anderes Bild. Die Dammkrone setzt sich um 6,3 m (Abbildung 6 (b)), was mehr als dem Vierfachen der Setzung bei mitteldichter Lagerung der Dammschüttung entspricht. Der Schwerpunkt der Verformungen liegt im wassergesättigten Bereich der Dammflanke unterhalb der Sickerlinie. Dort tritt mit 10,6 m eine sehr große horizontale Verschiebung auf, die zudem gegenüber der vertikalen Verschiebung $(1,3 \mathrm{~m})$ dominiert. Dies deutet auf ein Versagen der wasserseitigen Dammböschung hin. Die Ursache dessen liegt im Anstieg des Porenwasserdruckes begründet (Abbildung 7 (b)). Der Porenwasserüberdruck fällt an dem hier exemplarisch untersuchten Punkt A doppelt so hoch aus wie bei mitteldichter Lagerung, weil der locker gelagerte Sand eine höhere Neigung zur Verdichtung unter zyklischer Scherung aufweist. Dadurch wird die effektive Spannung fast vollständig abgebaut, der Boden steht kurz vor der Verflüssigung. Dementsprechend ist die Scherfestigkeit drastisch herabgesetzt, woraus schließlich die in Abbildung 6 (b) ersichtlichen großen Bodendeformationen folgen.

Die Ergebnisse belegen, dass mit Hilfe des CSSA-Modells die Auswirkungen zyklischer Beanspruchungen auf Erdbauwerke sinnvoll wiedergegeben werden können. Berechnungen dieser Art haben jedoch mehr als nur illustrativen Charakter. Sie sind in der Lage, das hochgradig nichtlineare Spannungs-Dehnungs-Verhalten des Bodens und den potentiell desaströsen Einfluss des Porenwassers auch bei komplexen Baugrund- und Grundwasserverhältnissen sowie Bauwerksgeometrien zu berücksichtigen. Daher sind solche Simulationen in der Praxis die Grundlage für eingehende Untersuchungen der Gebrauchstauglichkeit und der Standsicherheit, wenn empirische und analytische Bemessungsmodelle an ihre Grenzen stoßen.

\section{Potential für Weiterentwicklungen}

Das CSSA-Modell kann bereits wichtige Aspekte des Materialverhaltens von Sand unter zyklischer Beanspruchung abbilden. Trotzdem besteht noch Bedarf für Weiterentwicklungen.

Die Formulierung des CSSA-Modells fußt auf der Annahme infinitesimaler Verschiebungen und Verdrehungen. Bei vielen der eingangs genannten geotechnischen Fragestellungen ist diese Annahme jedoch nicht gerechtfertigt und verschlechtert die Qualität der Simulationsergebnisse, weil es zu großen Verformungen des Bodens im Berechnungsmodell kommen kann. Dies gilt 
insbesondere für Erdbebensimulationen und die numerische Modellierung der Pfahleinbringung. Außerdem kann es zu Konvergenzproblemen kommen, wenn Finite Elemente stark deformiert werden. In diesem Fall müsste ein modernes Simulationsverfahren benutzt werden, dass das FE-Netz auf mechanisch konsistente Weise entzerrt oder von vornherein netzfrei arbeitet. Vor diesem Hintergrund wurden in den letzten Jahren beispielsweise die Allgemeine Lagrange-Euler-Methode (ALE), die Multimaterielle Allgemeine Lagrange-Euler-Methode (MMALE), die Coupled-Euler-Lagrange-Methode (CEL) oder die Material-Point-Methode für die geotechnische Anwendung erschlossen [29]. Diese Verfahren werden aus der Theorie finiter Deformationen heraus entwickelt. Um kompatibel mit ihnen zu sein, muss das CSSA-Modell konsistent zur Theorie finiter Deformationen reformuliert werden.

Darüber hinaus gibt es Aspekte des Materialverhaltens, die noch nicht zufriedenstellend abgebildet werden können. So produziert das CSSA-Modell teils unrealistische Ergebnisse unter Bedingungen, bei denen große zyklische Änderungen der mittleren effektiven Spannung auftreten, wie beispielsweise beim Einrammen von Pfählen oder im Gründungsbereich von Schleusenbauwerken. Zur Illustration dient ein zyklischer Ödometerversuch mit Toyoura-Sand von Bauer [30]. Abbildung 8 zeigt die Spannungs-Porenzahl-Kurve des Versuchs und deren Nachrechnung unter Verwendung des Parametersatzes aus Tabelle 1. Im Laborversuch bewirken die Ent- und Wiederbelastungsschleifen der Schwelllast nur eine sehr geringe zusätzliche Verdichtung gegenüber der Erstbelastung. Das CSSA-Modell hingegen prognostiziert auch nach 20 Zyklen noch eine Verdichtung mit unverminderter Rate. Der Grund hierfür ist, dass die Verformungsgeschichte aus zyklischer Beanspruchung bisher nur im Kegelmechanismus des Modells angemessen berücksichtigt wird. Unter ödometrischen Verhältnissen ist jedoch vor allem der Kappenmechanismus relevant. Dessen Stoffgesetzfunktionen sind bisher nicht geeignet, den Einfluss zyklischer Effekte auf die Steifigkeit zu berücksichtigen. Um dieses Defizit zu beheben, sind zusätzliche Stoffgesetzfunktionen für die Verfestigungsregel der kappenförmigen Grenzfläche zu entwickeln. Hierfür muss zunächst das Verhalten von Sand bei zyklischer Kompression mit konstantem Spannungsverhältnis experimentell untersucht werden. 


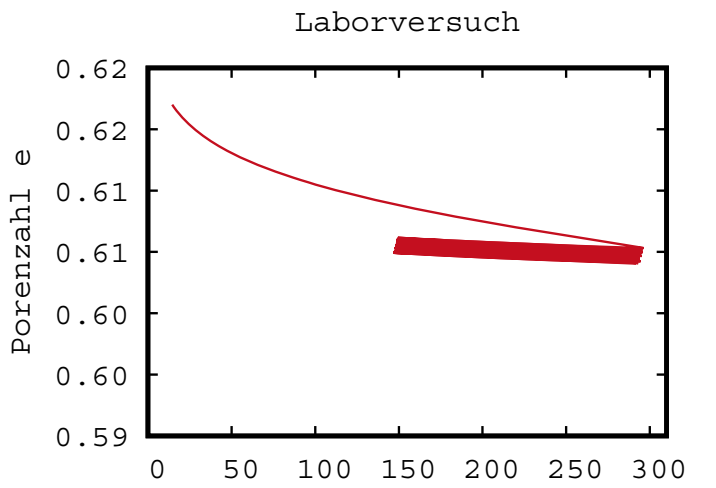

Simulation mit dem CSSA-Modell

effektive vertikale spannung $\sigma_{\mathrm{v}}$ in kPæffektive vertikale spannung $\sigma_{\mathrm{v}}$ in $\mathrm{kPa}$

Abbildung 8: Versuchsergebnis (links, aus [30]) und Nachrechnung (rechts) eines zyklischen Ödometerversuchs (Toyoura-

Sand, $e_{0}=0,617$ )

\section{Schlussfolgerungen und Ausblick auf zukünftige Arbeiten}

Der Beitrag zeigt auf, dass zur numerischen Lösung aktueller geotechnischer Problemstellungen Stoffgesetze für das Spannungs-Dehnungs-Verhalten des Bodens benötigt werden, die zyklische Lasten angemessen berücksichtigen. Einen sehr vielversprechenden Ansatz in Bezug auf nichtbindige Böden stellt das elastoplastische CSSA-Modell dar. Mit ihm lassen sich sowohl bei der Simulation monotoner und zyklischer Elementversuche als auch bei der Lösung komplexer Randwertaufgaben sehr gute Berechnungsergebnisse erzielen.

Zur Weiterentwicklung des CSSA-Modells werden aktuell und in naher Zukunft die folgenden Arbeiten ausgeführt:

- Reformulierung des Modells konsistent zur Theorie finiter Deformationen

- Durchführung von Laborversuchen zum Verhalten von Sand bei zyklischer Kompression

- Entwicklung zusätzlicher Stoffgesetzfunktionen für die kappenförmige Grenzfläche auf Grundlage der im vorigen Schritt experimentell gewonnenen Erkenntnisse, um zyklische Kompression mit der Kappe realistisch beschreiben zu können

- Validierung der Weiterentwicklungen, Erprobung des verbesserten Modells anhand der Nachrechnung geotechnischer Modell- und Feldversuche

Über die Arbeitsergebnisse wird in künftigen Veröffentlichungen berichtet werden.

\section{Danksagung}


Diese Arbeit entstand im Rahmen eines Forschungsvorhabens, das von der Deutschen Forschungsgemeinschaft über die Sachbeihilfe SA 310/27 gefördert wird. Für diese Unterstützung bedanken sich die Autoren. Außerdem sei den Gutachtern der Bautechnik für ihre konstruktiven

Hinweise zum Manuskript herzlich gedankt.

\section{Literatur}

[1] Triantafyllidis, T. (Hrsg.): Holistic Simulation of Geotechnical Installation Processes: Numerical and Physical Modelling, Lecture Notes in Applied and Computational Mechanics. 77 : Springer, 2015.

[2] von Wolffersdorff, P.-A.: A hypoplastic relation for granular materials with a predefined limit state surface. Mechanics of Cohesive-frictional Materials 1 (1996), H. 3, S. 251-271.

[3] Niemunis, A. ; Herle, I.: Hypoplastic model for cohesionless soils with elastic strain range. Mechanics of Cohesive-frictional Materials 2 (1997), S. 279-299.

[4] von Wolffersdorff, P.-A. ; Schwab, R.: The Uelzen I Lock - Hypoplastic finite-element analysis of cyclic loading. Bautechnik 86 (2009), H. S1, S. 64-73.

[5] Nübel, K. ; Cudmani, R.: Examples of finite element calculations with the hypoplastic law. In: Kolymbas, D. (Hrsg.): Constitutive Modelling of Granular Materials : Springer, 2000, S. 523-538.

[6] Fuentes, $W$. ; Triantafyllidis, Th. ; Lizcano, A.: Hypoplastic model for sands with loading surface. Acta Geotechnica 7 (2012), H. 3, S. 177-192.

[7] Wegener, D. ; Herle, I.: Prediction of permanent soil deformations due to cyclic shearing with a hypoplastic constitutive model. geotechnik 37 (2014), H. 2, S. 113-122.

[8] Niemunis, A. ; Grandas-Tavera, C. E.: Computer Aided Calibration, Benchmarking and Check-Up of Constitutive Models for Soils. Some Conclusions for Neohypoplasticity. In: Triantafyllidis, T. (Hrsg.): Holistic Simulation of Geotechnical Installation Processes: Theoretical Results and Applications. 82 : Springer, 2017, S. 168-192.

[9] Li, X. S. ; Dafalias, Y. F.: Dilatancy for cohesionless soils. Géotechnique 50 (2000), H. 4, S. 449-460.

[10] Schofield, A. N. ; Wroth, C. P.: Critical state soil mechanics. London u.a. : McGrawHill, 1968.

[11] $L i, X$. S.: A sand model with state-dependent dilatancy. Géotechnique 52 (2002), H. 3, S. $173-186$.

[12] Dafalias, Y. F.: Bounding Surface Plasticity. I: Mathematical Foundation and Hypoplasticity. Journal of Engineering Mechanics 112 (1986), H. 9, S. 966-987.

[13] Ming, H. Y. ; Li, X.-S.: Fully coupled analysis of failure and remediation of lower San Fernando Dam. Journal of Geotechnical and Geoenvironmental Engineering 129 (2003), H. 4, S. 336-349.

[14] $S u, D$. ; $L i, X$. S.: Impact of multidirectional shaking on liquefaction potential of level sand deposits. Géotechnique 58 (2008), H. 4, S. 259-267.

[15] Gao, Z. ; Zhao, J.: Constitutive Modeling of Anisotropic Sand Behavior in Monotonic and Cyclic Loading. Journal of Engineering Mechanics 141 (2015), H. 8

[16] Dafalias, Y. F. ; Manzari, M. T.: Simple Plasticity Sand Model Accounting for Fabric Change Effects. Journal of Engineering Mechanics 130 (2004), H. 6, S. 622-634.

[17] Taiebat, M. ; Jeremić, B. ; Dafalias, Y. F. ; Kaynia, A. M. ; Cheng, Z.: Propagation of seismic waves through liquefied soils. Soil Dynamics and Earthquake Engineering 30 (2010), H. 4, S. 236-257.

[18] Andrianopoulos, K. I. ; Papadimitriou, A. G. ; Bouckovalas, G. D.: Bounding surface plasticity model for the seismic liquefaction analysis of geostructures. Soil Dynamics and Earthquake Engineering 30 (2010), H. 10, S. 895-911.

[19] Boulanger, R. W. ; Ziotopoulou, K.: Formulation of a sand plasticity plane-strain model for earthquake engineering applications. Soil Dynamics and Earthquake Engineering 53 (2013), S. 254-267.

[20] Fuentes, $W$. ; Triantafyllidis, Th.: ISA model: A constitutive model for soils with yield surface in the intergranular strain space. International Journal for Numerical and Analytical Methods in Geomechanics 39 (2015), H. 11, S. 1235-1254.

[21] Taiebat, M. ; Dafalias, Y. F.: SANISAND: Simple anisotropic sand plasticity model. International Journal for Numerical and Analytical Methods in Geomechanics 32 
(2008), H. 8, S. 915-948.

[22] Prada Sarmiento, L. F.: Implementation of the elastoplastic Model Sanisand 2004 into Incremental Driver (Vortrag an der Universität Karlsruhe), 2009.

[23] Been, K. ; Jefferies, M. G.: A state parameter for sands. Géotechnique 35 (1985), H. 2, S. 99-112.

[24] Rackwitz, F.: Numerische Untersuchungen zum Tragverhalten von Zugpfählen und Zugpfahlgruppen in Sand auf der Grundlage von Probebelastungen, Technische Universität Berlin, Dissertation, 2003. - Veröffentlichungen des Grundbauinstitutes der Technischen Universität Berlin, Heft 32

[25] Seed, H. B. ; Lee, K. L. ; Idriss, I. M. ; Makdisi, F. I.: The Slides in the San Fernando Dams During the Earthquake of Febuary 9, 1971. Journal of the Geotechnical Engineering Division 101 (1975), H. 7, S. 651-688.

[26] Tasan, H. E. ; Rackwitz, F. ; Savidis, S. A.: Porenwasserdruckakkumulation bei zyklisch horizontal belasteten Monopiles mit großen Durchmessern. Bautechnik 87 (2010), H. 8, S. 449-461.

[27] Seekins, L. C. ; Brady, A. G. ; Carpenter, C. ; Brown, N.: Digitized Strong-Motion Accelerograms from North \& Central American Earthquakes 1933-1986, U.S. Geological Survey Digital Data Series ( Nr. DDS-7), 1992.

[28] Sze, H. Y. ; Yang, J.: Failure Modes of Sand in Undrained Cyclic Loading: Impact of Sample Preparation. Journal of Geotechnical and Geoenvironmental Engineering 140 (2014), H. 1, S. 152-169.

[29] Aubram, D.: Über die Berücksichtigung großer Bodendeformationen in numerischen Modellen. In: Herle, I. (Hrsg.): Ohde-Kolloquium 2014 - Aktuelle Themen der Geotechnik, 2014, S. 109-122.

[30] Bauer, E.: Zum mechanischen Verhalten granularer Stoffe unter vorwiegend ödometrischer Beanspruchung, Universität Karlsruhe, Dissertation, 1992. — Veröffentlichungen des Institutes für Bodenmechanik und Felsmechanik der Universität Fridericiana in Karlsruhe, Heft 130

\section{Autoren}

Christian Carow, M. Sc.

Technische Universität Berlin

Fachgebiet Grundbau und Bodenmechanik

Gustav-Meyer-Allee 25

13355 Berlin

christian.carow@tu-berlin.de

Prof. Dr.-Ing. Frank Rackwitz

Technische Universität Berlin

Fachgebiet Grundbau und Bodenmechanik

Gustav-Meyer-Allee 25

13355 Berlin

frank.rackwitz@tu-berlin.de

Prof. Dr.-Ing. Stavros Savidis

Technische Universität Berlin

Fachgebiet Grundbau und Bodenmechanik

Gustav-Meyer-Allee 25

13355 Berlin

savidis@tu-berlin.de 